death but did not differentiate patients who survived in a vegetative state from those who became normal. Cambining blood glucose and cerebral blood flow values improved predictability of outcome in near-drowning. (Ashwal $\mathrm{S}$ et al. Prognostic implications of hyperglycemia and reduced cerebral blood flow in childhood neardrowning. Neurology May 1990; $\underline{40: 820-823) .}$

COMMENT. Cerebral blood flow and blood glucose determinations on admission are useful predictors of the outcome of children with near-drowning and like the Glascow Coma Scale, they are objective measurements of prognosis.

\title{
CONGENITAL ANOMALIES
}

BRAIN ANOMALIES AND HEART DEFECTS

The type, frequency, and clinical presentation of developmental brain anomalies in 41 infants with the hypoplastic left heart syndrome are reviewed from the Children's Hospital of Philadelophia, and the University of Pennsylvania School of Medicine, Philadelphia. A major or minor central nervous system abnormality occurred in 29\% and included agenesis of the corpus callosum ( 3 cases), holoprosencephaly (1), micrencephaly (9), and cortical mantle malformation (8). Congenital brain anomalies were present in patients either with or without external dysmorphic features that involved the head, face and eyes, fingers, lung and kidney. In patients living only one day or less approximately $50 \%$ had recognizable malformations, microcephaly, and pulmonary anomalies. (Glauser TA et al. Congenital brain anomalies associated with the hypoplastic left heart syndrome.

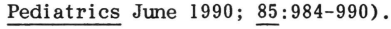

COMNENT. The authors recommend that infants with hypoplastic left heart syndrome deserve careful genetic, ophthalmologic and neurologic evaluations, including CT scan and long-term neurologic follow-up. In the same issue of Pediatrics the authors review their experience with acquired brain lesions associated with hypoplastic left heart syndrome, finding $45 \%$ with hypoxic, ischemic and hemorrhagic lesions secondary to aNS perfusion and glucose oxygen delivery. A duration of cardiopulmonary bypass with hypothermic total circulatory arrest longer than 40 minutes was associated with a higher incidence of acquired neuropathology. (Glauser TA et al. Acquired neuropathologic lesions associated with the hypoplastic left heart syndrome. Pediatrics June $1990 ;$ 85:991-1000).

New management strategies for perinatal hypoxic-ischemic encephalopathy (HIE) are reviewed by Vannucci RC. (Pediatrics June 1990; 85:961-967). Between 10\% and 60\% of asphyxiated neonates with HIE expire during the neonatal period. Of those who survive, $25 \%$ exhibit neuropsychologic deficits. 